THE COLIEGE OF AERONAUTICS

DEPARTMENT OF PRODUCTION AND INDUSTRIAI ADMINISTRATION

\title{
Flow along tool-chip interface \\ in orthogonal metal cutting
}

- by -

H.E. Enahoro* and P.I.B. OxIey*

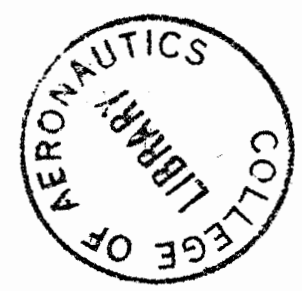

\section{S U M M A R}

In recent papers it has been suggested that over part of the toolchip contact zone the chip does not slide but sticks to the tool, chip flow taking place by shear within the body of the chip. Sticking contact is inconsistent with steady state cutting and in this paper a slip-line field model of chip flow is presented which does not include sticking contact and which is consistent with the relevant experimental observations.

"The College of Aeronautics, Cranfield. 


\section{INTRODUCTION}

In orthogonal cutting a surface layer of material is removed by a tool with a relative motion parallel to the machined surface and perpendicular to its own cutting eage. If the depth of cut ( $t$ in Fig. 1) is small compared to its width then deformation takes place under approximately plane strain conditions. If the chip (Fig. I) is formed by plastic deformation and there is no build up of material on the tool cutting edge the process is approximately a steady state process.

In analysing this process it has been usual to assume that the chip slides over the tool, but in recent papers Zorev (I) and Wallace and Boothroyd (2) have suggested that sliding only occurs over part of the contact (Fig. 2). Over the remainder of the contact it was assumed that sticking occurred with the layer of chip material in contact with the tool stationary relative to the tool and with chip flow resulting from shear within the chip. This model of chip flow was introduced to explain the following experimental observations: (a) over the sticking contact microscopic examination showed a deposit of chip material on the tool face; (b) the part of the chip in contact at the sticking contact had imprinted on it transverse scratches corresponding to those formed on the tool face in grinding; (c) photomicrographs of the chip (Fig. 3) showed that the layer of material adjacent to the sticking contact was retarded relative to the rest of the chip.

In our view the idea of sticking occurring over part of the toolchip contact length is inconsistent with steady state cutting and is 
apparently related to cutting with a built-up edge in which successive layers of chip material become stationary relative to the tool and build up to form a built-up edge. Tabor has put forward a similar suggestion in discussion of reference (2). However, it is known that the above observations, on which the sticking model of chip flow is based, can be made when cutting under approximately steady state conditions i.e. without a built-up edge. In what follows a model of chip flow for steady state cutting is given which is consistent with these observations and which does not lead to the difficulties introduced by assuming sticking to occur.

\section{Slip-Iine Field for Flow Along Tool-Chip Interface}

Slip-line fielas have been widely used in the analysis of two dimensional (plane strain) plasticity problems. The method is based on St. Venant's theory of plastic flow which assumes a plastic-rigid material in which elastic strains are neglected and the volume is assumed to remain constant during deformation. A slip-line field consists of two families of slip-lines which are orthogonal to each other and whose directions at any point represent the directions of maximum shear stress and maximum shear strain rate. It follows from the volume constancy assumption that the rate of extension along a slip-line is zero or in other words that two adjacent points in the plastic zone are on the same slip-line if their relative velocity cuts the line joining them at right angles. This latter property, the so called hodograph property of slip-lines, can be used to construct a slip-line field from the observed velocities of flow (see, for example, Palmer and Oxley (3)).

Using the hodograph method the slip-line field shown in Fig. 4 was

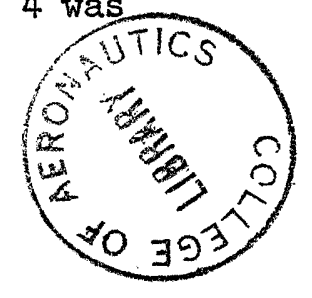


constructed for the flow along the tool-chip interface. The length of tool-chip contact and the radius of chip curvature used in the construction were obtained from a cine film taken of the side of a cut during orthogonal cutting, the cutting conditions being: depth of cut $=0.0045 \mathrm{in}$; width of cut $=0.25 \mathrm{in}$; cutting velocity $=0.5 \mathrm{in} / \mathrm{min}$; tool rake angle $=35^{\circ}$. The construction was carried out by a trial and error process, adjustments being made to the slip-line field until the following velocity boundary conditions were approximately satisfied: (I) material entering the plastic zone from the work and re-entering the work from the plastic zone had to have the velocity of the rigid work (i.e. cutting velocity); (2) material leaving the plastic zone and entering the chip had to have a velocity consistent with the rigid body rotation of the chip; (3) the llow adjacent to the tool face had to be in a direction parallel to the tool at the point considered. In addition it will be noted that a stagnation point of the flow occurs on the tool nose. Streamilnes above this point flow (eventually) into the chip while lower streamlines re-enter the work. The streamline meeting the stagnation point must have zero velocity at this point. For stress boundary conditions the only restraint placed on the construction was that the shear stress at the tool face should oppose the flow of material. At the stagnation point it was assumed that the material divides and that a very small load free surface exists adjacent to this point. For this reason the slip-lines were drawn at $45^{\circ}$ to the surface at the stagnation point with the hydrostatic stress equal to the shear flow stress and tensile. Palmer and Yeo (4) have considered this part of the flow some-

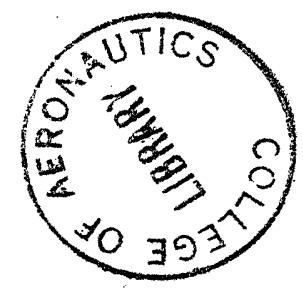


what differently and have suggested that a small triangular cap of rigid material exists in the region adjacent to the stagnation point. If such a cap of rigid material had been assumed in constructing the present slip-line field it would only have modified the flow, significantly, in the region of the stagnation point.

Using the hodograph a number of streamlines were constructed in the plastic zone (Fig. 4), the spacing between points representing the velocity. To show the form of the deformation the deformation of an initially rectangular grid as it passes through the slip-line field was constructed and is shown in Fig. 5.

\section{Discussion}

The streamline adjacent to the tool face (Fig. 4) shows that the velocity near the cutting edge is much slower than the velocity in the rigid part of the chip (as this streamline must leave the plastic zone with the corresponding velocity of the chip the flow accelerates along the tool face). This results in material adjacent to the cutting face being retarded relative to the rest of the chip. This retarded layer is clearly shown by the deformed grid in Fig. 5 which compares well with the photomicrograph (Fig. 3) of this region. This characteristic deformation was one of the observations which led to the introduction of sticking contact (1) (2) and it is important to note that even with relative sliding between chip and tool a retarded layer can occur.

To explain the relative sliding along the tool chip interface we can follow Tabor (5) and consider the surfaces to be separated by an interfacial 
film at their contact areas; this film consisting of contaminants and material from both the tool and chip. Flow occurs by shearing of this film and with this mechanism we would not be surprised to find chip material deposited on the tool face. In view of the high stresses at the tool-chip interface and the adjacent plastic zone in the chip it can be expected that the real area of contact will approach and in some cases be equal to the apparent area. Such a condition would not prevent sliding taking place by shearing of the interfacial film but would explain why the part of the chip in contact along the plastic zone has been observed to have scratches imprinted on it corresponding to those on the tool face. The model of chip flow given in Fig. 4 does not therefore appear to be inconsistent with any of the observations which led to the introduction of sticking contact. It has the advantage that it is a possible steady state model.

Above the plastic zone (Fig. 4) the curved chip will be stressed elastically and Hertzian flattening by the tool will occur. This zone probably corresponds to the sliding contact zone in references (1) and (2).

The slip line analysis presented in this paper is incomplete in that the field has not been checked for stress. Work is now in hand to check both the internal consistency of stress and to ensure that the stresses acting on the tool are consistent with the measured cutting forces. Variable flow stress slip-line theory (3) is being used in this part of the analysis. An attempt is also being made to check the theoretical streamlines of Fig. 4, experimentally. Cine films are being taken of this region (at a magnification of $x 60$ ) on the side of a workpiece which has been polished

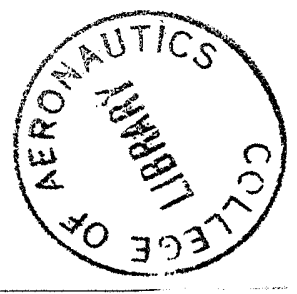


and etched. A plot of a preliminary film (made by tracing out the paths of individual grains) is given in Fig. 6 and it can be seen that reasonable agreement exists between the form of the experimental and theoretical streamlines.

\section{Acknowledgements}

We wish to acknowledge the many useful discussions with our colleague M.J.M. WeIsh.

\section{References}

1. Zorev, N.N.

2. Wallace, P.W. and Boothroyd, G. 'Tool forces and tool-chip friction in orthogonal machining .

Journal Mech. Eng. Sci., 1964, 6, 74.

3. Palmer, W.B. and Oxley, P.I.B. 'Mechanics of metal cutting'. Proc. I. Mech. E., I959, 173, 623.

4. Palmer, W.B. and Yeo, R.C.K. 'Metal flow near the tool point during orthogonal cutting with a blunt tool'. Proc. 4th Int. M.T.D.R. Conf. 1963, 61.

5. Tabor, $D$. 'Junction growth in metallic friction: the role of combined stresses and surface contamination.

Proc. Royal Soc., series A, 1959, 378. 